\title{
Das Jahrhundertprojekt der Nachhaltigkeit am Scheideweg:
}

\section{Wie kann die Energiewende in Deutschland breite gesellschaftliche Unterstützung finden?}

\section{Jörg Radtke}

Online publiziert: 16. April 2020

(C) Der/die Autor(en) 2020

\section{Quo vadis, Energiewende?}

Die Energiewende in Deutschland ist heute ein Transformationsvorhaben, das breite gesellschaftliche Anerkennung findet. Umfragen belegen, dass sie von einer überwältigenden Mehrheit der Bevölkerung als wünschenswert und sinnvoll angesehen wird $^{1}$. Das war nicht immer so. Als Bundeskanzlerin Angela Merkel unter dem unmittelbaren Eindruck der Fukushima-Reaktorkatastrophe 2011 die Energiewende ausrief, wirkte die dem beschlossenen Atomkraft-Ausstiegs zugrundeliegende Annahme, die Stromerzeugung wäre mittelfristig zu einem wesentlichen Anteil oder gar vollständig erneuerbar zu bewerkstelligen, sehr abstrakt. Können, so fragte man sich damals, die Erneuerbaren allein die Grundlast für die Industrie bereitstellen - und wäre nicht ganz Deutschland dann mit Windrädern und Photovoltaikanlagen übersät?

Auf diese erste Phase der Verunsicherung folgte eine Zeit wachsenden Vertrauens. Wie sich gegenwärtig zeigt, kann tatsächlich ein Großteil des Stroms durch Erneuerbare produziert werden. Hunderttausende Bürgerinnen und Bürger beteiligten sich am Ausbau der erneuerbaren Energien - ob durch eine eigene Photovoltaikanlage auf dem Dach oder in Form der Mitgliedschaft in einer Energiegenossenschaft. Der Windkraft-Ausbau an Land und vor der Küste schritt zügig voran. Die Energiewende, sie schien auf einem guten Weg.

\footnotetext{
1 Siehe die Umfragen der Agentur für Erneuerbare Energien: https://www.unendlich-viel-energie.de/ themen/akzeptanz-erneuerbarer/akzeptanz-umfrage/akzeptanzumfrage-2019 (letzter Zugriff: 22.03.2020).

J. Radtke $(\bowtie)$

Fakultät I/Politikwissenschaft, Universität Siegen, Adolf-Reichwein-Straße 2, 57068 Siegen,

Deutschland

E-Mail: radtke@ politikwissenschaft.uni-siegen.de
} 
Doch dann geriet die Energiewende in schwierigeres Fahrwasser. Mit dem Absenken der gesetzlich garantierten Einspeisevergütungen verlor die Photovoltaik an wirtschaftlicher Attraktivität, die vielbeachtete Bürgerenergie-Bewegung kam weitgehend zum Erliegen. Der lokale Widerstand gegen Windenergie und Biomasse wurde stärker, neue kritische Stimmen - aus Protestbewegungen, Naturschutzverbänden und politischen Parteien - stellten die Energiewende zunehmend in Frage. Der Strompreis stieg unterdessen weiter an, der Netzausbau erzeugte zusätzlichen Widerstand und krankt heute gleich dem Windkraft-Ausbau an langwierigen Verfahren. Derzeit befindet sich der Zubau an Windkraft an Land auf einem Tiefststand. Gleichzeitig wächst der Druck aufgrund der Klimaschutzziele und der politisch anvisierten flächendeckenden Umstellung auf elektrische Antriebe im Verkehr, die den Strombedarf massiv erhöhen wird.

So ist mittlerweile einiger Unmut entstanden. Hierbei wird kaum die Energiewende per se in Frage gestellt, die Kritik bezieht sich vielmehr auf das Management ${ }^{2}$. Die Energiewende sei aus dem Ruder gelaufen, Grad und Qualität der Umsetzung seien unzureichend, Kosten explodierten, ein Masterplan existiere nicht und überhaupt: es herrsche Chaos. Gleichzeitig diskutiert die Bundespolitik heftig über Windenergie, erwägt einerseits neue Förderkonzepte und andererseits strengere Abstandsregelungen, während immerhin dem Netzausbau fast einhellig dringender Beschleunigungsbedarf attestiert wird. Die Stimmung ,vor Ort“" ist wesentlich schwieriger einzufangen: Nach Ergebnissen des Sozialen Nachhaltigkeitsbarometers der Energiewende nimmt die Kritik vielerorts zu (Setton 2019). Grundsätzlich ist ob der Kontextualität die Bandbreite der Ablehnung sehr groß - sie reicht von friedlicher bis zu sehr feindlicher Energiewende-Kritik inklusive Morddrohungen gegen windkraftaffine Kommunalpolitikerinnen und -politiker.

Wenn indes im Wesentlichen Einigkeit darüber besteht, dass die Energie- und Verkehrswende vorangetrieben werden müssen, wenn also der Ausbau der Erneuerbaren zum politischen Konsens wird und erhebliche Eingriffe und Maßnahmen (das heißt auch: Umstellungen) in der Folge erfordert - was kann dann politisch getan werden, um die Energiewende als „Gemeinschaftswerk“ (Ethik-Kommission Sichere Energieversorgung 2011) mit breiter gesellschaftlicher Akzeptanz umzusetzen?

\section{Was ist Akzeptanz - und wie verhält sie sich zur Legitimation?}

In gesellschaftlichen und politischen Debatten zur Energiewende wird vielfach auf die Akzeptanz verwiesen, die ein solch einschneidender sozio-technischer Regimewechsel sowohl von Seiten der Bevölkerung als auch der organisierten Interessen erfordere (Mast und Stehle 2016; Schmalz 2019). Dahinter steht in der Regel die Annahme, dass Akzeptanz auch politische Legitimation bedeute bzw. damit gleichzusetzen sei, wie es Niklas Luhmann unmissverständlich formuliert hatte (Luhmann 1969, 2007). Diese Verquickung weist zwei grundsätzliche Schwachpunkte auf: Erstens bleibt zumeist unklar, was Akzeptanz im Einzelfall genau bedeuten soll (Meint sie die aktive Unterstützung eines potenziell strittigen Unterfangens? Handelt es sich

\footnotetext{
2 Siehe etwa den einschlägigen Cover-Beitrag des SPIEGELS (Dohmen et al. 2019).
} 
im Sinne der Toleranz eher um ein neutrales Hinnehmen? Oder genügt das Ausbleiben von Widerstand, um Akzeptanz zu konstatieren?), zweitens blendet sie andere Formen der demokratischen Erzeugung von Legitimation aus (Sareen 2019).

Oftmals wird die Akzeptanz der Energiewende daran bemessen, wie viele der an einer Repräsentativität beanspruchenden Umfrage Teilnehmenden - etwa Bürgerinnen und Bürger - angeben, die Nutzung von Windenergie etc. zu befürworten (s. FN1). Die Aussagekraft solcher allgemeinen Einstellungsabfragen ist begrenzt: So äußern sich Befragte gegenüber Energiewende-Projekten, die in räumlicher Nähe zu ihrem Wohnort realisiert werden (sollen), deutlich kritischer (Bertsch et al. 2016; Hübner et al. 2019; Setton 2019). Akzeptanz ist einzelfallbezogen, abstrakte Stimmungsbilder scheinen zu ihrer Bestimmung nur eingeschränkt geeignet. Schließlich ist entscheidend, worauf sich die Akzeptanz beziehen soll: Ist es die Techniknutzung als solche (Output), geht es um die räumliche Verteilung, also die Standorte von Anlagen (distributive Dimension) oder um die dazugehörigen Planungen, Verfahren und Abläufe (prozessuale Dimension/Throughput)? Weiterhin können der Grad der Berücksichtigung örtlicher Gemeinschaften und Gegebenheiten (Anerkennungsdimension), der individuelle oder kollektive Einbezug (Input) und der Impact dieser Beteiligung auf das Ergebnis (Outcome) wesentliche Auswirkungen auf die Akzeptanz zeitigen. Analog dazu werden in der Politikwissenschaft klassisch drei Dimensionen der Legitimation unterschieden: Input, Output und Throughput (Scharpf 1999). Im Rahmen von Untersuchungen zur Akzeptanz und Partizipation im Kontext der Energiewende wurden Defizite hinsichtlich aller drei Dimensionen identifiziert (Hildebrand et al. 2017). Insbesondere der inhaltlich-prozessualen Qualität von Beteiligungsverfahren (Prinzipien der Ausgewogenheit, Fairness, Transparenz, Verhältnismäßigkeit) wird eine entscheidende Bedeutung für die Akzeptanz beigemessen (Suškevičs et al. 2019). Zweifelsohne ist an diesen (potentiell) legitimationsstiftenden Funktionen nicht zu rütteln. Allerdings zeigt ein Blick in die historische Beteiligungsforschung, dass die zahlreichen Schwachstellen der Energiewende-Partizipation und Governance sowie der einschlägigen politischen Willensbildungsprozesse letztlich nichts Neues sind - ältere Untersuchungen lesen sich mitunter wie Blaupausen des Scheiterns zeitgenössischer Beteiligungs- und Entscheidungsprozesse (statt vieler: Habermas 1973).

Immer öfter akzeptieren die Rechtsadressaten das prozedural legitimierte Recht nicht mehr, sodass eine Legitimationskrise angenommen werden kann (Römmele und Schober 2013). Vor diesem Hintergrund scheint es vielversprechend, Konzepte demokratischer Willens-, Konsens- und Legitimationsbildung neu zu diskutieren und ggf. zu erweitern. Pierre Rosanvallon elaboriert neue Formen demokratischer Legitimität, da er das alte System der doppelten Legitimität ${ }^{3}$ schwinden sieht (Rosanvallon 2013, S. 78). Die neue Ära der Partikularität zeitigt ein dezentriertes System, das neue legitimatorische Mechanismen einer Entscheidungs- und Verhal-

\footnotetext{
3 Das alte System kennzeichnet sich durch Einsetzungs- und Identifikationslegitimität - also einerseits die gesellschaftliche Anerkennung der Regierungsgewalt sowie andererseits die Identifikation mit Normen und Werten (Rosanvallon 2013, S. 11).
} 
tensdemokratie in Gang setzt ${ }^{4}$, welche sich durch Qualitäten konstituieren, die immer wieder neu verhandelt werden müssen (ebd., S. 7, 15, 24). Rosanvallon stellt daher drei neue Legitimitäten vor - Unparteilichkeit, Reflexivität und Nähe -, die eine Verbindung zwischen den klassischen Prinzipien der Legitimation und der gesellschaftlichen Wahrnehmung herstellen (ebd., S. 15). Auf Verfahren der Energiewende sind die drei Prinzipien gut übertragbar, bedürfen jedoch der näheren definitorischen Bestimmung. Auch die kritische Auseinandersetzung mit Energiewende-Konflikten und Partizipation kann für die Bestimmung von Akzeptanz und Legitimation zielführend sein: Die Kritik an Top-down-Ausrichtungen von Diskurs und Verfahren sowie die Frage des Zulassens, d.h. des (konstruktiven) Austragens von Konflikten, führen zu neuen Bewertungen und Maßstäben (Cuppen 2018; Kühne 2018; Weber 2018), wie sie etwa im Rahmen der Radikalen Demokratie diskutiert werden (Comtesse et al. 2019; Marchart 2020). Auch die Ansätze der Deliberation, auf welche viele Verfahren im Kern setzen, stehen bzgl. stark divergierender Interessen, Grenzen der Konsensbildung und oftmals engen Entscheidungsspielräumen erneut auf dem Prüfstand (Bächtiger und Parkinson 2019; Curato et al. 2019; Elstub et al. 2018; Niessen 2019).

\section{Eine Frage der Steuerung? Energiewende-Governance zwischen Mehrebenen- und Komplexitätsproblematik}

Die Energiewende-Governance hat ein Janusgesicht. Einerseits zeigt sie - auch historisch bedingt - stark zentralisierte Züge, andererseits ist die Energiewende grundsätzlich ein dezentrales Unterfangen, denn Maßnahmen werden vor Ort umgesetzt und lokale Institutionen verfügen dabei durchaus über Einfluss (Benz und Czada 2019). Eine Governance mit langfristiger Planungsperspektive (Kamp 2016) findet sich in einem beinahe unauflösbaren Spannungsfeld wieder: Tagesaktuelle Dynamiken in Zeiten der Globalisierung und Day-to-day-Politics stehen einerseits der fokussierten Ausrichtung auf ein gesamtgesellschaftliches Ziel-Szenario fundamental entgegen. Andererseits machen die Klimaschutz-Ziele eine langfristige Ausrichtung alternativlos. Es handelt sich hierbei um ein Dilemma, an dem Politik und Staat nahezu scheitern müssen (Holgeid und Thompson 2013). Die Energiewende droht, sich in den Dynamiken zeitgenössischer Problemlösungsstrategien zu verfangen. Uwe Schimank (2019) verweist in diesem Zusammenhang auf die gesellschaftlichen Komplexitäten, die funktional differenzierte und kapitalistische Ordnung sowie erhöhte Legitimationserfordernisse und Globalisierungsdruck - mithin zahlreiche unkalkulierbare Faktoren, die Steuerung erschweren.

\footnotetext{
4 Die beiden neuen Formen ergeben sich aus der Unvereinbarkeit der indirekten Demokratie und der Sphäre der Entscheidungen (Rosanvallon 2013, S. 21), denn es besteht ein Widerspruch zwischen ,Anerkennung der Legitimität des Konflikts und dem Streben nach Konsens“ sowie zwischen ,,realistische[m] Entscheidungsprinzip (der Mehrheit) und einem notwendigerweise anspruchsvolleren Rechtfertigungsprinzip (Einstimmigkeit)“ (ebd.: S. 21 f.). Dieses Spannungsverhältnis verdeutlicht die Fiktionalität der zugrundeliegenden Prinzipien (Gleichsetzung der Mehrheit mit der Einstimmigkeit), welche bislang verleugnet und kaschiert worden sei (ebd.: S. 23). Neu ist insbesondere die Verhaltensdemokratie, denn das Verhalten der Regierenden hat sich im „Bewusstsein der Bürger sehr stark bemerkbar gemacht“ (ebd.: S. 24).
} 
Neben der Problematik gleichzeitiger Erforderlichkeit und Unmöglichkeit langfristiger Planung (Kamp 2016) wird die Steuerung der Energiewende durch Föderalismus und Mehrebenen-Problematik erschwert (Müller und Kahl 2015; Schreurs und Steuwer 2015; Wurster und Köhler 2016). Christine Chemnitz (2018) erklärte den Energiewende-Konsens zu einem Mythos, da bei näherer Betrachtung wenig Übereinstimmung bei Zielen, Maßnahmen und Strategien im Detail existiert (Metapher der sechszehn verschiedenen Energiewenden der Bundesländer). Schon im Jahr 1972 beschrieb Fritz Scharpf die ,Komplexität als Schranke der politischen Planung". Er arbeitete die Probleme der Politikverflechtung mit den typischen Verflechtungsfallen dezidiert aus (Scharpf et al. 1976; Scharpf 1993; Mayntz und Scharpf 1995). Solche Koordinationsprobleme lassen sich auch in der Energiewende finden. Aktuell argumentiert hingegen Arthur Benz (2019), dass Verteilungskonflikte der Energiewende nicht durch die etablierte föderale Politikverflechtung lösbar sind und man daher darauf verzichte. Stattdessen setze man auf Verhandlungen auf Regierungsebene und stärke die zentrale Bundesebene. Die Steuerungsprobleme der Energiewende in Form von Koordinationsdefiziten und institutioneller Fragmentierung seien daher auf einen Mangel an Verflechtung zwischen Bund und Ländern zurückzuführen, eine Abstimmung aller Ebenen indes zwingend erforderlich. Die Diagnose ist zwar zutreffend, jedoch brächte eine stärkere Verflechtung womöglich andere Problemlagen mit sich. Christian Bauer (2015) konnte für den Netzausbau zeigen, dass die Abstimmung zwischen allen Trägern öffentlicher Belange im Beteiligungsverfahren der Bundesnetzagentur an Partizipationsverflechtungsfallen scheiterte, da eine Harmonisierung aller Interessen schlechterdings nicht möglich ist und stattdessen Unverständnis und Frustration entstehen (vgl. auch Fink und Ruffing 2018, 2020).

Roland Czada (2019) verweist zunächst nüchtern auf typische Reaktionsmuster im Angesicht von Konsenshürden und Koordinationsproblemen: Per Muddling Through werde fallweise und situativ flexibel reagiert - was kein neues Phänomen darstelle. Problematisch seien aber der Verlust stabiler Mehrheiten und korporatistischer Steuerungsformen sowie die Zerfaserung der Interessenvermittlung durch unzählige Gremien. Eben solche Kommissionen sind für heterogene Multi-AkteursKonstellationen eigentlich das geeignete Mittel im Sinne einer vermittelnden dritten Ebene, wie etwa Paul Sabatier (1993) argumentierte. Doch die Kohle-Kommission verdeutlicht, woran auch diese Vermittlungslösungen scheitern können: sie stellen zumeist eher einen „Schein-Konsens“ her, der die unterschiedlichen Interessenlagen politischer Ebenen und Akteure nicht miteinander versöhnt - und nach Beendigung der Kommissionsarbeit geht das Gezerre weiter. Allerdings sind im Kontext der Energiewende Herausforderungen zu bewältigen, die, im Vergleich zum alten Energiesystem mit einer sehr begrenzten Anzahl handelnder Akteure, durch zahlreiche, sich teils überlagernde, teils diametral gegenüberstehende sowie in dynamischen Entwicklungen befindliche Interessenlagen geprägt sind, was nicht nur die Vermittlung zwischen den Akteuren, sondern auch ihre eigenen Handlungsentscheidungen maßgeblich erschwert (Neukirch 2019). Die Herausforderungen für die Verhandlungsdemokratie sind also immens - ein Blick auf die langfristigen Reform- bzw. Wendeprojekte vergangener Jahrzehnte zeigt, dass die Energiewende hierbei durchaus eine Sonderrolle einnimmt (Czada und Radtke 2018). 
Forderungen nach einem Masterplan für die Energiewende (Dohmen et al. 2019) ist aus zwei Gründen kritisch zu begegnen: Die Bund-Länder-Verhandlungen zeigen, dass eine Verschiebung von Verantwortlichkeiten in Richtung des Bundes und - damit einhergehend - ein höherer Grad an Zentralisierung unrealistisch sind; überdies könnte ein solcher Masterplan nichts an den einschlägigen rechtlichen Regelungen (z. B. Immissionsschutz-, Bau- und Planungsrecht sowie insbesondere am europäischen Recht), Planungsgrundsätzen sowie den föderalen Grundprinzipien ändern. Hinzu kommen noch die Abhängigkeiten vom Marktgeschehen. So ist zu konstatieren: Heterogene Ausprägungen der Energiewende - Windkraft an der Küste, Biomasse in Agrarregionen - sind unausweichliche Realitäten, die nicht in Einklang gebracht werden können. Daher kommt es in entscheidendem Maße auf ein kluges Ausgleichs- und Kompensationsmanagement neben schonender Implementierung an.

So wird eine erhebliche Herausforderung zukünftig in der Ausbildung und Verschärfung regionaler Disparitäten und Stadt-Land-Differenzen liegen (Holstenkamp und Radtke 2019; Rodden 2019): Ambitionierte und häufig sozioökonomisch bessergestellte Städte und Regionen entwickeln sich zu Leuchttürmen des Klimaschutzes, während marginalisierte und deprivierte Provinzen zurückstehen, in denen Entwicklungen wie Windenergie-Ausbau und Kohleausstieg zusätzlich unmittelbare negative Auswirkungen auf Einstellungen in der Bevölkerung haben können. Hier gilt es, Ausgleichsmechanismen und Aufwertungsstrategien zu entwickeln, die der immerhin grundgesetzlich festgeschriebenen Gleichwertigkeit der Lebensverhältnisse dienen (Spatial und Territorial Justice: Bouzarovski und Simcock 2017; Degros et al. 2019; vgl. auch Bojarra-Becker et al. 2016; Erbguth 2019).

\section{Eine Frage der Beteiligung? Ein fragwürdiger Königsweg}

Die Energiewende ist ein partizipatives Transformationsprojekt und damit maßgeblich geprägt durch die Beteiligung von Einzelpersonen, unterschiedlichen Gemeinschaften und diversen Akteuren (Holstenkamp und Radtke 2018). Hierbei sind unterschiedliche Dimensionen zu unterscheiden: Eine materielle und assoziative Beteiligungsvariante durch den vollständigen oder anteiligen Erwerb von Energieanlagen bzw. Infrastruktur, eine diskursiv-deliberative Beteiligung im Rahmen formeller und informeller Öffentlichkeitsbeteiligung sowie eine partizipative Governance-Gestaltung (Radtke und Renn 2019). Ergänzend kommen noch Elemente der direkten Demokratie wie Bürgerentscheide und Abstimmungen hinzu (Biegelbauer und Kapeller 2017). Damit verbindet die Beteiligung im Rahmen der Energiewende bürgerschaftliches Engagement in Form von Bürgerenergie und diversen Bürgerinitiativen (Radtke et al. 2020) mit dialogisch oder deliberativ geprägten Verfahren, die vor allem bei Planungsverfahren und der Entwicklung von Energiekonzepten Anwendung finden (Renn et al. 2017).

Die Beteiligung wird weithin als Königsweg und wesentlicher Schlüssel zur Erreichung der angestrebten Akzeptanz und Legitimation gesehen. Diese „Panakeia“ der Energiewende (Schweizer und Renn 2013), diese „silver bullet“ für die Akzeptanz 
(Rau et al. 2012) gestaltet sich jedoch weder unkompliziert noch voraussetzungsfrei ${ }^{5}$. Beteiligung setzt erstens die Bereitschaft voraus, die Beeinflussung von Willensbildungsprozessen auch tatsächlich zuzulassen (Output/Outcome), zweitens muss sie der gesamten (lokalen) Bevölkerung bzw. allen tangierten Akteuren zur Verfügung stehen, besser noch: sie involvieren (Input) und drittens muss der Prozess bestimmte Qualitätskriterien (Offenheit, Fairness, Transparenz etc.) erfüllen (Throughput) auch im Sinne der Maßstäbe von Nähe, Unparteilichkeit und Reflexivität nach Rosanvallon. Im Lichte dieser Anforderungen werden diverse Mängel der Partizipation in der Energiewende offenbar, derer hier nur einige angeführt werden können.

Die materielle Beteiligung in Gestalt der Bürgerenergie hat ihre Bestzeiten wohl hinter sich, seit Jahren gründen sich nur noch wenige Initiativen. Immerhin konnten in etwa 1700 Beteiligungsgesellschaften viele tausend Bürgerinnen und Bürger beteiligt werden (Kahla et al. 2017). Allerdings zeigt ein Blick auf die Sozialstruktur der Involvierten, dass vor allem Menschen aus sozioökonomisch bessergestellten Teilen der Bevölkerung Mitglied werden, auch jüngere Personen und Frauen partizipieren weniger (Radtke 2016). Zudem werden viele Bürgerenergie-Projekte mit lokalem Bezug nur von einem Bruchteil der Ortsansässigen realisiert, wodurch soziale Spaltungen begünstigt werden. Kommunale Betreibungsmodelle, in denen eine Gemeinde im Auftrag der lokalen Bevölkerung handelt, erscheinen weniger exklusiv (Radtke 2018).

Auch ein Blick auf die Öffentlichkeitsbeteiligung, die vor allem beim Windkraftund Netzausbau ausgiebig praktiziert wird, offenbart zahlreiche Herausforderungen und Defizite. Trotz aufwendiger und weitreichender Bemühungen öffentlicher Einrichtungen und privater Vorhabenträger herrscht auf Seiten derjenigen, die an Arbeitsgruppen, Dialogforen und Informationsveranstaltungen teilnehmen, viel Enttäuschung und Frustration. Dies ist auf stark divergierende Rationalitäten, wenig Spielraum für Verhandlungen und die Selbstwahrnehmung als in besonderem Maße benachteiligt, die sich bedingt durch Systemlogiken bei allen Beteiligten beobachten lässt, zurückzuführen (Huge und Roßnagel 2018; Komendantova und Battaglini 2016; Leibenath et al. 2016; Roßmeier et al. 2018; Kapeller und Biegelbauer 2020). So plädieren Bürgerinitiativen bei Netzausbau-Beteiligungsverfahren für eine dezentrale Energiewende, die den Ausbau in Teilen mutmaßlich überflüssig machen würde - eine Forderung, die für die Planerinnen und Planer sowie die Netzbetreiber kaum eine probate Diskussionsgrundlage darstellt (Neukirch 2017). In den Veranstaltungen aufkommende Fragen und Anliegen übersteigen oftmals die Kompetenzen der zuständigen Behörden, worin sich die Mehrebenen-Governance-Problematik widerspiegelt. Wenngleich die Beteiligungsverfahren im Zuge des Netzausbaus eine negative Bilanz aufweisen (Kamlage et al. 2014; Schweizer-Ries et al. 2016) können durch faire und offene Verfahren, welche nicht primär durch den Anspruch einer möglichst zügigen und kosteneffizienten Realisierung des Projekts geprägt sind, konfliktlösende Potentiale entfaltet werden (Kamlage et al. 2020). Obschon Defizite den Involvierten bekannt sind, bleibt das partizipative Paradigma mit den üblichen Ansätzen der Infomessen und Dialogformate angesichts fehlender Alternativen in der

\footnotetext{
${ }^{5}$ Siehe kritisch hierzu: Lorenz et al. (2019); Merkel (2015); Rhodius (2012); Ritzi (2014); Ritzi und Merkel (2017); Roth (2016); Schmalz (2019); van Deth (2014) sowie Vetter und Remer-Bollow (2017).
} 
Praxis und ungebrochen - oder umgekehrt ausgedrückt: Verzicht auf Partizipation ist mit dem Zeitgeist unvereinbar geworden. Nach wie vor ist auf interaktionaler Ebene in den Beteiligungsverfahren eine implizite Top-down-Systematik inklusive einer abdämpfend wirkenden paternalistisch-positivistischen Kulisse vielerorts erkennbar, die jedoch zunehmend kritisch hinterfragt wird (Cuppen 2018).

Damit werden die Ansprüche an Partizipation der Zivilgesellschaft und partizipative Governance auf eine Probe gestellt. Plädoyers für eine stärker konsultative Rückkopplung zwischen den etablierten politischen Entscheidungsstrukturen und diversen bürgerorientierten Beteiligungsformaten sind zwar berechtigt und sinnvoll, vermögen allerdings an den schwerlich zu harmonisierenden Divergenzen und Eigenlogiken bzw. -motivationen der einzelnen Interessenträger wenig zu ändern (Nanz und Leggewie 2016; Römmele und Schober 2013).

\section{Fazit: Die Energiewende am Scheideweg}

Die Energiewende ist einerseits eine Erfolgsgeschichte: Als im Jahr 2000 das Erneuerbare-Energien-Gesetz im Bundestag verabschiedet wurde, war nicht absehbar, dass nur zwanzig Jahre später immerhin die Stromwende als erfolgreich eingeleitet gelten kann. Die tiefgreifenderen Transformationsdesiderate einer Verkehrs- und Wärmewende sowie einer vollends vollzogenen Stromwende, die gänzlich auf erneuerbare Erzeugung setzt, werden jedoch weitaus schwieriger zu erreichen sein - und sehr viel erheblichere Einschnitte bedeuten (Paul Welfens spricht daher zu Recht von einem „Ende der Komfortzone“ in der Klimaschutzpolitik, vgl. Welfens 2019).

Dies zeigt sich erstens an der gegenwärtigen Debatte um die Windkraft. Der stagnierende Windkraft-Ausbau hat verschiedene Ursachen. In vielen Regionen sind Regelungen und Planungsverfahren so aufwendig, restriktiv und unsicher sowie/oder durch Klageverfahren eingeschränkt, dass entweder Standorte nicht erschlossen werden können oder die Risiken durch mögliche Betreiber als zu groß wahrgenommen werden. In der Politik werden verschiedene Vorschläge diskutiert, etwa Quoten für den Ausbau oder Prämien für Anwohnerinnen und Anwohner. Hierbei ist zu bedenken: Selbst wenn Mindestabstände verringert und Vergütungen erhöht würden, hätte dies kaum Auswirkungen auf die Geschwindigkeit der Verfahren - sie zögen sich auch weiterhin jahrelang hin. Da nunmehr erstmals Energieproduktionsanlagen wie PV und Windkraft aus der garantierten Förderung nach dem EEG nach 20 Jahren herausfallen werden, stellt sich die Frage, ob und inwieweit diese auch weiterhin betrieben werden (können) - zumal noch eine bereits nahezu ausgeschöpfte Deckelung der Photovoltaik-Förderung gültig ist, um die neben den Abstandsregelungen im Rahmen der Energiewende-Verhandlungen derzeit in den Bund-Länder-Verhandlungen gerungen wird (Stand April 2020). Und zu bedenken ist etwa im Falle eines möglichen Repowerings von Windkraft, dass nach Ablauf der vorgesehenen Betriebszeit eine Neubewertung der Standorte durchgeführt wird - also Fälle denkbar sind, in denen Windenergieanlagen nicht ersetzt werden können. All das stellt in Aussicht, dass der anvisierte weitere Ausbau der Erneuerbaren vor enormen Herausforderungen steht. 
Zweitens rückt der Diskurs in den Mittelpunkt (Mast und Stehle 2016; Rosenberger und Kleinberger 2017). Hier ist ein zunehmender Einfluss von Populismus und einer aufgeheizten Debattenkultur zu beobachten (FSB 2020; Radtke et al. 2019): Bei zahlreichen Energiewende-Konfliktherden vor Ort verschärft sich der Diskurs und enthemmt sich der Sprachduktus (Eichenauer et al. 2018). Klimaskeptizismus und kritischen Politik- und Staatsauffassungen kommt hier eine entscheidende Rolle zu (Brunnengräber 2018; Forchtner 2019; Radtke und Schreurs 2019); mitunter speist sich die Ablehnung gar aus negativen Beteiligungserfahrungen (Eichenauer 2018). Hierbei kommt jenseits simplifizierender NIMBY-Deutungsversuche Gerechtigkeitsfragen eine besondere Relevanz zu (Energy Justice: Williams und Doyon 2019). Zudem lassen sich Stellvertreter-Konflikte beobachten, im Zuge derer Verhältnisse zwischen Stadt und Land, Alteingesessenen und Zugezogenen, Eigentum und Nicht-Eigentum kontrovers diskutiert werden (Fettke 2018; Hoeft et al. 2017). In Zukunft wird die räumliche Gerechtigkeit zwischen marginalisierten und sozioökonomisch sowie infrastrukturbezogen besser gestellten Regionen bzw. Städten vermutlich eine sehr viel wichtigere Rolle spielen.

Drittens werden Herausforderungen durch die internationale und europäische Energie- und Klimapolitik entstehen: Die EU versucht durch Konturierung einer europäischen Energiepolitik an Einfluss zu gewinnen - bislang vor allem mit weichen Regelungsmechanismen (Oberthür 2019). Dies könnte sich zukünftig ändern, wenn der Green New Deal ernst genommen wird, und würde zu Änderungen und Anpassungserfordernissen der deutschen Energiepolitik führen. Bereits jetzt sind neue Nachhaltigkeitsstrategien in Folge der Reformen der europäischen Vergaberechtsvorschriften zu erwarten (Ziekow und Gyulai-Schmidt 2020).

Schließlich ist viertens eine erhebliche Wirkmächtigkeit der Zivilgesellschaft und Sozialer Bewegungen wie Fridays for Future zu beobachten, die auch zukünftig eine ambitionierte Klimapolitik einfordern werden. Zwar zeigt sich neben einer gewissen Frustration auch ein neues Bewusstsein für die Unabwendbarkeit des Klimawandels (Franzen 2020). Dies ist aber nicht zwingend mit Resignation gleichzusetzen, sondern könnte in Zukunft noch stärker das zivilgesellschaftliche Engagement aktivieren. Die globale Corona-Krise zeigt auf, welche Potentiale für den Klimaschutz durch Verzicht existieren - da der Lockdown-Zustand aber moderne Gesellschaften nicht trägt, sind neue und solidarische Konzepte für die Nachhaltigkeitstransformation wichtiger denn je.

Mit Blick auf die zukünftig erforderliche breite Akzeptanz im Sinne grundsätzlicher Zustimmung zu den Vorhaben der Energiewende sind (idealtypisch) zwei Entwicklungen denkbar: Entweder kommt es zu einer „Versöhnung“ mit den diversen Eingriffen und Belastungen, neuen Routinen sowie Konsum- sowie Mobilitätsmustern oder es entsteht ob der persistierenden Widerstände ein ,stilles“ Abkommen zwischen den Mehrheiten, das nur moderate oder sogar keine weiteren Maßnahmen vorsieht - als Analogon zu ,gefühlten“ Mehrheiten, auf die Politik reagiert (Renn 2019).

Es ist unwahrscheinlich, dass eine der beiden Optionen in der beschriebenen Reinform realisiert wird. Allerdings ist zu vermuten, dass politische Entscheidungsträgerinnen und -träger erhebliche, d. h. stark belastende und einschneidende Konzepte einer radikaleren Transformation nicht verfolgen werden. Die antizipativ-reaktive 
Politik ist anfällig für Stimmungen, die bereits gegenwärtig Geschwindigkeitsbegrenzungen für Autobahnen nicht mehrheitsfähig machen. Die von Axel Berg ins Spiel gebrachte Idee, die „Energiewende einfach durchzusetzen“, wirkt auf den ersten Blick wie eine naheliegende Möglichkeit, die Realisierung der Energiewende zu beschleunigen. Dies geht jedoch an jeder staatsrechtlichen Realität vorbei und entspricht darüber hinaus nicht den Grundsätzen der Demokratie (Berg 2019). Demokratisch wird eben nichts einfach umgesetzt, sondern aufwendig und langwierig ausgehandelt und immer wieder neu verhandelt - ein schwieriger und doch lohnender Weg, obgleich die Uhr des Klimawandels ticken mag.

Zur Erhöhung und Sicherstellung von Akzeptanz und Legitimität werden Politik und Staat auch weiterhin auf Beteiligung und situativ-flexible Energiewende-Strategien sowie bestenfalls reflexive Konzepte setzen, was wohl im Kern der richtige Ansatz ist. Jenseits einer Auslagerung von Debatten an Bürgerforen wären Änderungen innerhalb der Institutionen, die mehr Input und Reflexivität zur Legitimitätserhöhung erlaubten (Meinel 2019; SRU 2019), sowie eine Reformierung von Planungsrecht und -praxis für eine besser abgestimmte Koordination und Steuerung zu erwägen (Heitzmann 2018; Kümper 2018; Schmid et al. 2020). Dies sind allerdings dicke Bretter, die es zu bohren gälte. In Verbindung mit der Digitalisierung sind vielversprechende einschlägige Zukunftsformate bereits erkennbar (WBGU 2019) - nun braucht es reflexive, ausgewogene und intelligente Nutzungskonzepte, um die Potentiale einer digitalen Demokratie auch in der Energiewende besser auszuschöpfen.

Funding Open Access funding provided by Projekt DEAL.

Open Access Dieser Artikel wird unter der Creative Commons Namensnennung 4.0 International Lizenz veröffentlicht, welche die Nutzung, Vervielfältigung, Bearbeitung, Verbreitung und Wiedergabe in jeglichem Medium und Format erlaubt, sofern Sie den/die ursprünglichen Autor(en) und die Quelle ordnungsgemäß nennen, einen Link zur Creative Commons Lizenz beifügen und angeben, ob Änderungen vorgenommen wurden.

Die in diesem Artikel enthaltenen Bilder und sonstiges Drittmaterial unterliegen ebenfalls der genannten Creative Commons Lizenz, sofern sich aus der Abbildungslegende nichts anderes ergibt. Sofern das betreffende Material nicht unter der genannten Creative Commons Lizenz steht und die betreffende Handlung nicht nach gesetzlichen Vorschriften erlaubt ist, ist für die oben aufgeführten Weiterverwendungen des Materials die Einwilligung des jeweiligen Rechteinhabers einzuholen.

Weitere Details zur Lizenz entnehmen Sie bitte der Lizenzinformation auf http://creativecommons.org/ licenses/by/4.0/deed.de.

\section{Literatur}

Bächtiger, André, und John Parkinson. 2019. Mapping and measuring deliberation: towards a new deliberative quality. New York: Oxford University Press.

Bauer, Christian. 2015. Die Energiewende in der Politik- und Partizipationsverflechtungsfalle: Das Beispiel des Stromnetzausbaus. Verwaltungsarchiv 106:112-154.

Benz, Arthur. 2019. Koordination der Energiepolitik im deutschen Bundesstaat. dms - der moderne staat - Zeitschrift für Public Policy, Recht und Management 12(2):299-312. https://doi.org/10.3224/dms. v12i2.01.

Benz, Arthur, und Roland Czada. 2019. Politische Steuerung von Transformation - das Beispiel der Energiepolitik. dms - der moderne staat - Zeitschrift für Public Policy, Recht und Management 12(2):243-250. https://doi.org/10.3224/dms.v12i2.05. 
Berg, Axel. 2019. Energiewende einfach durchsetzen: Roadmap für die nächsten 10 Jahre. München: Oekom.

Bertsch, Valentin, Margeret Hall, Christof Weinhardt, und Wolf Fichtner. 2016. Public acceptance and preferences related to renewable energy and grid expansion policy: empirical insights for Germany. Energy 114:465-477. https://doi.org/10.1016/j.energy.2016.08.022.

Biegelbauer, Peter, und Sandro Kapeller. 2017. Mitentscheiden oder Mitgestalten: Direkte Demokratie versus dialogorientierte Verfahren in lokalen Entscheidungsfindungsprozessen. SWS-Rundschau 57:32-55.

Bojarra-Becker, Elke, Klaus J. Beckmann, Rainer Danielzyk, Peter Dehne, Markus Eltges, Heike Köckler, Anne Ritzinger, Gerd Schäde, Jan Matthias Stielike, und Alexandra Tautz. 2016. Daseinsvorsorge und gleichwertige Lebensverhältnisse neu denken: Perspektiven und Handlungsfelder. Positionspapier aus der ARL. Hannover: Verlag der ARL, Akademie für Raumforschung und Landesplanung.

Bouzarovski, Stefan, und Neil Simcock. 2017. Spatializing energy justice. Energy Policy 107:640-648. https://doi.org/10.1016/j.enpol.2017.03.064.

Brunnengräber, Achim. 2018. Klimaskeptiker im Aufwind. In Bausteine der Energiewende, Hrsg. Olaf Kühne, Florian Weber, 271-292. Wiesbaden: Springer VS. https://doi.org/10.1007/978-3-65819509-0_13.

Chemnitz, Christine. 2018. Der Mythos vom Energiewendekonsens. Ein Erklärungsansatz zu den bisherigen Koordinations- und Steuerungsproblemen bei der Umsetzung der Energiewende im Föderalismus. In Energiewende. Politikwissenschaftliche Perspektiven, Hrsg. Jörg Radtke, Norbert Kersting, 155-203. Wiesbaden: Springer VS. https://doi.org/10.1007/978-3-658-21561-3_6.

Comtesse, Dagmar, Oliver Flügel-Martinsen, Franziska Martinsen, und Martin Nonhoff. 2019. Radikale Demokratietheorie: Ein Handbuch. Berlin: Suhrkamp.

Cuppen, Eefje. 2018. The value of social conflicts. Critiquing invited participation in energy projects. Energy Research \& Social Science 38:28-32. https://doi.org/10.1016/j.erss.2018.01.016.

Curato, Nicole, Marit Hammond, und John B. Min. 2019. Power in deliberative democracy. Norms, Forums, Systems. New York: Springer.

Czada, Roland. 2019. Politikwenden und transformative Politik in Deutschland. dms - der moderne staat - Zeitschrift für Public Policy, Recht und Management 12(2):400-417. https://doi.org/10.3224/dms. v12i2.12.

Czada, Roland, und Jörg Radtke. 2018. Governance langfristiger Transformationsprozesse. Der Sonderfall „Energiewende“. In Energiewende. Politikwissenschaftliche Perspektiven. Schriftenreihe Energietransformation, Hrsg. Jörg Radtke, Norbert Kersting, 45-75. Wiesbaden: Springer VS. https://doi. org/10.1007/978-3-658-21561-3_3.

Degros, Aglaée, Eva Schwab, und Daniel Gethmann. 2019. GAM 15: territorial justice. Berlin: Jovis Berlin.

van Deth, Jan W. 2014. Das schwierige Verhältnis zwischen Partizipation und Demokratie. In Mehr Partizipation - mehr Demokratie, Hrsg. Kerstin Pohl, Peter Massing, 9-21. Schwalbach: Wochenschau Verl.

Dohmen, Frank, Alexander Jung, Stefan Schultz, und Gerald Traufetter. 2019. Grüner Blackout. Der Spiegel 19: 12-21.

Eichenauer, Eva. 2018. Energiekonflikte - Proteste gegen Windkraftanlagen als Spiegel demokratischer Defizite. In Energiewende. Politikwissenschaftliche Perspektiven, Hrsg. Jörg Radtke, Norbert Kersting, 321-347. Wiesbaden: Springer VS. https://doi.org/10.1007/978-3-658-21561-3_11.

Eichenauer, Eva, Fritz Reusswig, Lutz Meyer-Ohlendorf, und Wiebke Lass. 2018. Bürgerinitiativen gegen Windkraftanlagen und der Aufschwung rechtspopulistischer Bewegungen. In Bausteine der Energiewende, Hrsg. Olaf Kühne, Florian Weber, 633-651. Wiesbaden: Springer VS. https://doi.org/10. 1007/978-3-658-19509-0_32.

Elstub, Stephen, Selen A. Ercan, und Ricardo Fabrino Mendonca. 2018. Deliberative systems in theory and practice. London: Routledge.

Erbguth, Wilfried. 2019. Zur Gleichwertigkeit der Lebensverhältnisse: rechtliche Grundlagen und raumplanerische Steuerungsmöglichkeiten. Umwelt- und Planungsrecht 39:483-494.

Ethikkommission Sichere Energieversorgung der Bundesregierung. 2011. Deutschlands Energiewende: Ein Gemeinschaftswerk für die Zukunft. Berlin: Die Bundesregierung.

Fettke, Ulrike. 2018. Etablierte und Außenseiter in der Kommunalpolitik? Eine Fallstudie zu Windkraft in einer badenwürttembergischen Kleinstadt. SOI Discussion Paper 2018-03. Stuttgart: Universität Stuttgart.

Fink, Simon, und Eva Ruffing. 2018. Öffentlichkeitsbeteiligung durch private Akteure: Eine empirische Analyse des deutschen Verfahrens zur Erstellung von Netzentwicklungsplänen. In Energiewende. 
Politikwissenschaftliche Perspektiven, Hrsg. Jörg Radtke, Norbert Kersting, 245-267. Wiesbaden: Springer VS. https://doi.org/10.1007/978-3-658-21561-3_8.

Fink, Simon, und Eva Ruffing. 2020. Learning in iterated consultation procedures - the example of the German electricity grid demand planning. Utilities Policy. Virtual Special Issue: Energy Transition Europe. (im Erscheinen).

Forchtner, Bernhard (Hrsg.). 2019. The Far Right and the Environment. Politics, Discourse and Communication. London: Routledge. https://doi.org/10.4324/9781351104043.

Forschungsjournal Soziale Bewegungen (FSB). 2020. Klima und Zivilgesellschaft. Forschungsjournal Soziale Bewegungen (1). (im Erscheinen).

Franzen, Jonathan. 2020. Wann hören wir auf, uns etwas vorzumachen?: Gestehen wir uns ein, dass wir die Klimakatastrophe nicht verhindern können. Hamburg: Rowohlt.

Habermas, Jürgen. 1973. Legitimationsprobleme im Spätkapitalismus. Frankfurt am Main: Suhrkamp.

Heitzmann, Daniel. 2018. Raumplanung und Energie: Planungsrechtliche Möglichkeiten zur Entwicklung der Energieinfrastruktur. Wien: Verlag Österreich.

Hildebrand, Jan, Irina Rau, und Petra Schweizer-Ries. 2017. Höhere öffentliche Akzeptanz durch bessere Beteiligungsverfahren? UVP-Report 31: 269-273.

Hoeft, Christoph, Sören Messinger-Zimmer, und Julia Zilles. 2017. Bürgerproteste in Zeiten der Energiewende. Lokale Konflikte um Windkraft, Stromtrassen und Fracking. Bielefeld: transcript.

Holgeid, Kjetil, und Mark Thompson. 2013. A reflection on why large public projects fail. In The governance of large-scale projects linking citizens and the state, Hrsg. Andrea Römmele, Henrik Schober, 219-243. Baden-Baden: Nomos.

Holstenkamp, Lars, und Jörg Radtke (Hrsg.). 2018. Handbuch Energiewende und Partizipation. Wiesbaden: Springer VS.

Holstenkamp, Lars, und Jörg Radtke. 2019. Stadt-Land-Disparitäten in der Energiewende. In Dezentral, partizipativ und kommunikativ: Zukunft der Energiewende, Hrsg. Jan-Hendrik Kamlage, Steven Engler, 137-176. Nordhausen: Traugott Bautz.

Hübner, Gundula, Johannes Pohl, Jan Warode, Boris Gotchev, Patrizia Nanz, Dörte Ohlhorst, Michael Krug, Steven Salecki, und Wolfgang Peters. 2019. Naturverträgliche Energiewende. Akzeptanz und Erfahrungen vor Ort. Bonn: Bundesamt für Naturschutz.

Huge, Antonia, und Alexander Roßnagel. 2018. Möglichkeiten der Öffentlichkeitsbeteiligung in Planungsund Genehmigungsverfahren von Windenergieanlagen. In Handbuch Energiewende und Partizipation, Hrsg. Lars Holstenkamp, Jörg Radtke, 613-625. Wiesbaden: Springer VS.

Kahla, Franziska, Lars Holstenkamp, Jakob R. Müller, und Heinrich Degenhart. 2017. Entwicklung und Stand von Bürgerenergiegesellschaften und Energiegenossenschaften in Deutschland. Lüneburg: Leuphana Univ.

Kamlage, Jan-Hendrik, Emily Drewing, Julia Lena Reinermann, Nicole de Vries, und Marissa Flores. 2020. Fighting fruitfully? Participation and conflict in the context of electricity grid extension in Germany. Utilities Policy 64:101022. https://doi.org/10.1016/j.jup.2020.101022.

Kamlage, Jan-Hendrik, Patrizia Nanz, und Björn Fleischer. 2014. Dialogorientierte Bürgerbeteiligung im Netzausbau. In Im Brennpunkt: Die Energiewende als gesellschaftlicher Transformationsprozess 4. Jahrbuch Nachhaltige Ökonomie., Hrsg. Hans-Christoph Binswanger, Felix Ekardt, Anja Grothe, Wolf-Dieter Hasenclever, Ingomar Hauchler, Martin Jänicke, Karl Kollmann, Nina V. Michaelis, Hans G. Nutzinger, Holger Rogall, und Gerhard Scherhorn, 195-216. Marburg: Metropolis.

Kamp, Georg (Hrsg.). 2016. Langfristiges Planen. Zur Bedeutung sozialer und kognitiver Ressourcen für nachhaltiges Handeln. Berlin: Springer.

Kapeller, Sandro, und Peter Biegelbauer. 2020. How (not) to solve local conflicts around alternative energy production: six cases of siting decisions of Austrian wind power parks. Utilities Policy 64. (im Erscheinen).

Komendantova, Nadejda, und Antonella Battaglini. 2016. Beyond Decide-Announce-Defend (DAD) and Not-in-My-Backyard (NIMBY) models? Addressing the social and public acceptance of electric transmission lines in Germany. Energy Research \& Social Science 22:224-231. https://doi.org/10. 1016/j.erss.2016.10.001.

Kühne, Olaf. 2018. Neue Landschaftskonflikte - Überlegungen zu den physischen Manifestationen der Energiewende auf der Grundlage der Konflikttheorie Ralf Dahrendorfs. In Bausteine der Energiewende, Hrsg. Olaf Kühne, Florian Weber, 163-186. Wiesbaden: Springer VS. https://doi.org/10.1007/ 978-3-658-19509-0_8.

Kümper, Boas. 2018. Querschnittsprobleme des Umwelt- und Planungsrechts: Rechtsschutz und Umweltprüfungen. Deutsches Verwaltungsblatt 133:1055-1057. https://doi.org/10.1515/dvbl-20181331606. 
Leibenath, Markus, Peter Wirth, und Gerd Lintz. 2016. Just a talking shop? - Informal participatory spatial planning for implementing state wind energy targets in Germany. Utilities Policy 41:206-213. https:// doi.org/10.1016/j.jup.2016.02.008.

Lorenz, Astrid, Christian P. Hoffmann, und Uwe Hitschfeld (Hrsg.). 2019. Partizipation für alle und alles? Fallstricke, Grenzen und Möglichkeiten. Wiesbaden: VS.

Luhmann, Niklas. 1969. Legitimation durch Verfahren. Neuwied: Luchterhand.

Luhmann, Niklas. 2007. Politische Planung: Aufsätze zur Soziologie von Politik und Verwaltung. 5. Aufl. Wiesbaden: VS.

Marchart, Oliver. 2020. Der demokratische Horizont: Politik und Ethik radikaler Demokratie. Berlin: Suhrkamp.

Mast, Claudia, und Helena Stehle. 2016. Energieprojekte im öffentlichen Diskurs. Erwartungen und Themeninteressen der Bevölkerung. Wiesbaden: Springer VS.

Mayntz, Renate, und Fritz W. Scharpf (Hrsg.). 1995. Gesellschaftliche Selbstregelung und politische Steuerung. Frankfurt am Main: Campus.

Meinel, Florian. 2019. Vertrauensfrage: Zur Krise des heutigen Parlamentarismus. München: C.H.Beck.

Merkel, Wolfgang. 2015. Nur schöner Schein? Demokratische Innovationen in Theorie und Praxis. Frankfurt am Main: Otto-Brenner-Stiftung.

Müller, Thorsten, und Hartmut Kahl (Hrsg.). 2015. Energiewende im Föderalismus. Baden-Baden: Nomos.

Nanz, Patrizia, und Claus Leggewie. 2016. Die Konsultative. Mehr Demokratie durch Bürgerbeteiligung. Berlin: Wagenbach.

Neukirch, Mario. 2017. Die Dynamik des Konflikts um den Stromtrassenbau: Stabilität, Wandel oder Stagnation? SOI Discussion Paper. Stuttgart: Universität Stuttgart.

Neukirch, Mario. 2019. Transition of energy systems: patterns of stability and change. In Handbook of energy governance in Europe, Hrsg. Michèle Knodt, Jörg Kemmerzell, 1-29. Cham: Springer. https:// doi.org/10.1007/978-3-319-73526-9_40-1.

Niessen, Christoph. 2019. When citizen deliberation enters real politics: how politicians and stakeholders envision the place of a deliberative mini-public in political decision-making. Policy Sciences 52:481-503. https://doi.org/10.1007/s11077-018-09346-8.

Oberthür, Sebastian. 2019. Hard or soft governance? The EU's climate and energy policy framework for 2030. Politics and Governance 7:17-27. https://doi.org/10.17645/pag.v7i1.1796.

Radtke, Jörg. 2016. Bürgerenergie in Deutschland. Partizipation zwischen Rendite und Gemeinwohl. Wiesbaden: Springer VS. https://doi.org/10.1007/978-3-658-14626-9.

Radtke, Jörg. 2018. Energiedemokratie durch Bürgerenergie? Die Grenzen finanzieller Bürgerbeteiligung. GAIA-Ecological Perspectives for Science and Society 27:284-286. https://doi.org/10.14512/gaia. 27.3.7.

Radtke, Jörg, und Ortwin Renn. 2019. Partizipation und bürgerschaftliches Engagement in der Energiewende. In Energiewende. Eine sozialwissenschaftliche Einführung, Hrsg. Jörg Radtke, Weert Canzler, 283-316. Wiesbaden: Springer VS. https://doi.org/10.1007/978-3-658-26327-0_10.

Radtke, Jörg, und Miranda Schreurs. 2019. Klimaskeptizismus und populistische Bewegungen in Europa und den USA. In Energiewende in Zeiten des Populismus, Hrsg. Jörg Radtke, Weert Canzler, Miranda Schreurs, und Stefan Wurster, 145-179. Wiesbaden: Springer VS. https://doi.org/10.1007/978-3658-26103-0_5.

Radtke, Jörg, Weert Canzler, Miranda Schreurs, und Stefan Wurster. 2019. Energiewende in Zeiten des Populismus. Wiesbaden: Springer VS. https://doi.org/10.1007/978-3-658-26103-0.

Radtke, Jörg, Emily Drewing, Eva Eichenauer, Lars Holstenkamp, Jan-Hendrik Kamlage, Franziska Mey, Jan Warode, und Jana Wegener. 2020. Energy transition and civic engagement. In The role of public participation in energy transitions, Hrsg. Ortwin Renn, Frank Ulmer, und Anna Deckert, 81-91. Cambridge: Academic Press. https://doi.org/10.1016/B978-0-12-819515-4.00004-0.

Rau, Irina, Jan Hildebrand, und Elke Weingarten. 2012. Akzeptanz durch Beteiligungsverfahren beim Netzausbau: Möglichkeiten und Grenzen des rechtlichen Rahmens. UVP-Report 26:166-175.

Renn, Ortwin. 2019. Gefühlte Wahrheiten. Orientierung in Zeiten postfaktischer Verunsicherung, 2. Aufl., Leverkusen: Barbara Budrich.

Renn, Ortwin, Wolfgang Köck, Pia-Johanna Schweizer, Jana Bovet, Christina Benighaus, Oliver Scheel, und Regina Schröter. 2017. Öffentlichkeitsbeteiligung bei Planungsverfahren der Energiewende. In Die Energiewende verstehen - orientieren - gestalten: Erkenntnisse aus der Helmholtz-Allianz. ENERGY-TRANS, Hrsg. Jens Schippl, Armin Grunwald, und Ortwin Renn, 547-567. Baden-Baden: Nomos.

Rhodius, Regina. 2012. Mehr Legitimität? Zur Wirksamkeit partizipativer Verfahren in räumlichen Planungsprozessen. Freiburg: Universität Freiburg. 
Ritzi, Claudia. 2014. Legitimitätsgenerierung im deliberativen und repräsentativen Paradigma: Komplementär oder kompetitiv? In Deliberative Demokratie in der Diskussion, Hrsg. Claudia Landwehr, Rainer Schmalz-Bruns, 239-270. Baden-Baden: Nomos.

Ritzi, Claudia, und Wolfgang Merkel. 2017. Die Legitimität direkter Demokratie: wie demokratisch sind Volksabstimmungen? Wiesbaden: Springer VS.

Rodden, Jonathan A. 2019. Why cities lose: the deep roots of the urban-rural political divide. New York: Basic Books.

Römmele, Andrea, und Henrik Schober. 2013. How to link citizens and the state: reasons for-and first steps towards - a participatory mode of governance. In The governance of large-scale projects, Hrsg. Andrea Römmele, Henrik Schober, 11-25. Baden-Baden: Nomos.

Rosanvallon, Pierre. 2013. Demokratische Legitimität. Unparteilichkeit - Reflexivität - Nähe. Bonn: Bundeszentrale für Politische Bildung.

Rosenberger, Nicole, und Ulla Kleinberger (Hrsg.). 2017. Energiediskurs: Perspektiven auf Sprache und Kommunikation im Kontext der Energiewende. Bern: Peter Lang.

Roßmeier, Albert, Florian Weber, und Olaf Kühne. 2018. Wandel und gesellschaftliche Resonanz - Diskurse um Landschaft und Partizipation beim Windkraftausbau. In Bausteine der Energiewende, Hrsg. Olaf Kühne, Florian Weber, 653-679. Wiesbaden: Springer VS. https://doi.org/10.1007/978-3-65819509-0_33.

Roth, Roland. 2016. Mehr Beteiligung bedeutet weniger Demokratie. Ein unlösbares politisches Paradoxon in der aktuellen Beteiligungsdebatte? In Politik mit Bürgern - Politik für Bürger Praxis und Perspektiven einer neuen Beteiligungskultur, Hrsg. Manuela Glaab, 59-74. Wiesbaden: Springer VS.

Sabatier, Paul A. 1993. Advocacy-Koalitionen, Policy-Wandel und Policy-Lernen: Eine Alternative zur Phasenheuristik. In Policy-Analyse. Kritik und Neuorientierung, Hrsg. Adrienne Héritier, 116-148. Opladen: Westdeutscher Verlag.

Sachverständigenrat für Umweltfragen (SRU).. 2019. Demokratisch regieren in ökologischen Grenzen Zur Legitimation von Umweltpolitik. Berlin: SRU.

Sareen, Siddharth (Hrsg.). 2019. Enabling sustainable energy transitions: practices of legitimation and accountable governance. Cham: Palgrave Pivot.

Scharpf, Fritz W. 1993. Positive und negative Koordination in Verhandlungssystemen. In Policy-Analyse. Kritik und Neuorientierung, Hrsg. Adrienne Héritier, 57-83. Opladen: Westdeutscher Verlag.

Scharpf, Fritz W. 1999. Regieren in Europa: effektiv und demokratisch? Frankfurt am Main: Campus.

Scharpf, Fritz Wilhelm, Bernd Reissert, und Fritz Schnabel. 1976. Politikverflechtung: Theorie und Empirie des kooperativen Föderalismus in der Bundesrepublik. Kronberg: Scriptor.

Schimank, Uwe. 2019. Politische Gesellschaftsgestaltung heute - sozial- und gesellschaftstheoretische Erwägungen angesichts von Transformationsvorhaben wie der „Energiewende“. dms - der moderne staat - Zeitschrift für Public Policy, Recht und Management 12(2):251-265. https://doi.org/10.3224/ dms.v12i2.03.

Schmalz, Inkeri Märgen. 2019. Akzeptanz von Großprojekten: Eine Betrachtung von Konflikten, Kostenund Nutzenaspekten und Kommunikation. Wiesbaden: VS.

Schmid, Benedikt, Christian Schulz, und Sabine Weck. 2020. Postwachstum und Raumentwicklung. Keimzellen für die Transformation. politische ökologie 160:19-26.

Schreurs, Miranda A., und Sibyl Steuwer. 2015. Der Koordinierungsbedarf zwischen Bund und Ländern bei der Umsetzung der Energiewende aus politikwissenschaftlicher Sicht. In Energiewende im Föderalismus, Hrsg. Thorsten Müller, Hartmut Kahl, 45-67. Baden-Baden: Nomos.

Schweizer, Pia-Johanna, und Ortwin Renn. 2013. Partizipation in Technikkontroversen: Panakeia für die Energiewende? TATuP - Zeitschrift für Technikfolgenabschätzung in Theorie und Praxis 22(2): 42-47. https://doi.org/10.14512/tatup.22.2.42.

Schweizer-Ries, Petra, Jan Hildebrand, Maximilian Hinse, Silke Rühmland, und Irina Rau. 2016. Begleitund Akzeptanzforschung zu aktuellen Fragen des Stromnetzausbaus in Deutschland: Wissenschaftliche Begleitung der Planungspraxis (Akzeptanz Netzausbau). Projektabschlussbericht. Saarbrücken: IZES. https://doi.org/10.2314/GBV:872386333.

Setton, Daniela. 2019. Soziales Nachhaltigkeitsbarometer der Energiewende 2018. Potsdam: IASS. https:// doi.org/10.2312/iass.2019.002.

Suškevičs, M., S. Eiter, S. Martinat, D. Stober, E. Vollmer, C.L. de Boer, und M. Buchecker. 2019. Regional variation in public acceptance of wind energy development in Europe: what are the roles of planning procedures and participation? Land Use Policy 81:311-323. https://doi.org/10.1016/j. landusepol.2018.10.032.

Vetter, Angelika, und Uwe Remer-Bollow. 2017. Bürger und Beteiligung in der Demokratie. Eine Einführung. Wiesbaden: Springer VS. 
WBGU - Wissenschaftlicher Beirat der Bundesregierung Globale Umweltveränderungen. 2019. Unsere gemeinsame digitale Zukunft. Berlin: WBGU.

Weber, Florian. 2018. Von der Theorie zur Praxis - Konflikte denken mit Chantal Mouffe. In Bausteine der Energiewende, Hrsg. Olaf Kühne, Florian Weber, 187-206. Wiesbaden: Springer VS. https://doi. org/10.1007/978-3-658-19509-0_9.

Welfens, Paul J. J. 2019. Klimaschutzpolitik - Das Ende der Komfortzone. Neue wirtschaftliche und internationale Perspektiven zur Klimadebatte. Wiesbaden: Springer. https://doi.org/10.1007/978-3-65827884-7.

Williams, Stephen, und Andréanne Doyon. 2019. Justice in energy transitions. Environmental Innovation and Societal Transitions 31:144-153. https://doi.org/10.1016/j.eist.2018.12.001.

Wurster, Stefan, und Christina Köhler. 2016. Die Energiepolitik der Bundesländer: Scheitert die Energiewende am deutschen Föderalismus? In Die Politik der Bundesländer: zwischen Föderalismusreform und Schuldenbremse, 2. Aufl., Hrsg. Achim Hildebrandt, Frieder Wolf, 283-314. Wiesbaden: Springer VS.

Ziekow, Jan, und Andrea Gyulai-Schmidt (Hrsg.). 2020. Nachhaltigkeitsstrategien im Zuge der Modernisierung der europäischen Vergaberechtsvorschriften. Berlin: Duncker \& Humblot. 